\title{
Pharmaceutical cannabinoid use in Manitoba, 2004/05 to 2014/15: a population-based cross-sectional study
}

\author{
Wajd Alkabbani BSc (Pharm), Ruth Ann Marrie MD PhD, Shawn Bugden MSc PharmD, \\ Silvia Alessi-Severini PhD, Paul Daeninck MD, James Bolton MD, Jitender Sareen MD, \\ Christine Leong BSc (Pharm) PharmD
}

\section{Abstract}

Background: Pharmaceutically derived cannabinoids are used for several indications, particularly pain management. The extent of their use from a population perspective is unknown; hence, the aim of this study was to evaluate trends in pharmaceutical cannabinoid use in Manitoba.

Methods: This was a retrospective population-based cross-sectional study using administrative data from the Manitoba Centre for Health Policy. Pharmaceutical cannabinoid users residing in Manitoba from Apr. 1, 2004, to Mar. 31 , 2015 were identified. We assessed the annual prevalence and incidence of pharmaceutical cannabinoid use, and the sociodemographic characteristics and medical conditions of users.

Results: We identified 5181 people who received at least 1 prescription for a pharmaceutical cannabinoid over the study period, 5033 of whom received their first prescription after Apr. 1, 2004. Nabilone accounted for 73650 (96.0\%) of all prescriptions dispensed; dronabinol was discontinued during the study period. The annual prevalence rate of use increased by $527.2 \%$, from $21.5(95 \%$ confidence interval $[\mathrm{Cl}$ 21.4-21.6) users per 100000 people in 2004/05 to $134.9(95 \% \mathrm{Cl} 134.7-135.1)$ users per 100000 people in $2014 / 15$. The annual incidence rate increased by $413.3 \%$, from 12.1 (95\% Cl 12.1-12.2) users per 100000 person-years in $2004 / 05$ to 62.2 (95\% Cl 62.1-62.4) users per 100000 person-years in 2014/15. The highest use was among older adults aged 46-64 years, females and urban area residents. One-third of incident users (1775 [35.3\%]) had a diagnosis of fibromyalgia in a 2-year period before their first cannabinoid prescription. General practitioners initiated almost half (2350 [46.7\%]) of first prescriptions, and anesthesiologists/pain specialists initiated one-quarter (1299 [25.8\%]).

Interpretation: The prevalence and incidence of pharmaceutical cannabinoid use increased over time. These findings provide insight into the use of cannabinoids before the introduction of recreational marijuana, which may affect this trend.

annabis is widely used in Canada: $42.5 \%$ of Canadians aged 15 years or more report having used cannabis in their lifetime, and $17.7 \%$ of Canadians who used cannabis reported using it for medical purposes. $^{1,2}$ Although cannabis has a long history of being used for several conditions such as pain and epilepsy, there is a lack of high-level evidence to support its use. ${ }^{3-5}$ Pharmaceutically derived prescription cannabinoids include nabilone, dronabinol and nabiximols; currently only nabilone and nabiximols are available in Canada. ${ }^{6}$ The use of these agents must now be considered in light of the recreational cannabis legalization enacted in Canada in October 2018, a move that has garnered critical attention regarding the potential economic, social and public health implications. ${ }^{7-9}$ One particular concern is the blurring of recreational and medical use, which has been available since 2001, and the potential confusion in health care monitoring of those using it medically. Pharmaceutically prepared cannabinoids may provide better dosage standardization and administration consistency compared to raw cannabis when used for therapeutic purposes. Moreover, pharmaceutical cannabinoids pose a lower risk of abuse compared to plant-based cannabis. ${ }^{10-12}$ Nabilone is a cannabinoid receptor agonist approved in 1982 in Canada for chemotherapy-induced

\section{Competing interests: None declared.}

This article has been peer reviewed.

Correspondence to: Christine Leong, christine.leong@umanitoba.ca CMAJ Open 2018. DOI:10.9778/cmajo.20180109 
nausea and vomiting not responding to conventional therapy. Dronabinol, an oral form of tetrahydrocannabinol, was approved in 1994 for chemotherapy-induced nausea and vomiting and for anorexia associated with HIV and AIDS but was discontinued in 2012. Nabiximols, an extract containing tetrahydrocannabinol and cannabidiol, was approved in 2005 for central neuropathic pain in multiple sclerosis and in 2007 for intractable cancer pain. These medications have also appeared in practice guidelines as third-line alternatives for several off-label indications, such as fibromyalgia and arthritis. ${ }^{13-15}$ Several studies have investigated the potential efficacy and possible adverse effects of pharmaceutical cannabinoids; $;^{3,16-18}$ however, there is still a lack of information regarding their use in the population. An understanding of the extent of use in the real world can be achieved by studies that rely on analyzing drug dispensations and physician claims, collected prospectively for health care system management. Characterizing the past trends of cannabinoid use enables a review of their use and establishes baseline measurements before recreational cannabis legalization. Therefore, we analyzed the prevalence and incidence of pharmaceutical cannabinoid use in Manitoba, a Canadian province with a relatively stable population of 1316424 as of 2015 . We also determined the demographic characteristics and medical conditions of users.

\section{Methods}

\section{Data sources}

We conducted a retrospective population-based crosssectional study from Apr. 1, 2004, to Mar. 31, 2015 using data obtained from the administrative databases within the Manitoba Population Research Data Repository located at the Manitoba Centre for Health Policy, University of Manitoba. ${ }^{19}$ This repository captures the encounters with the health care system for more than $98 \%$ of the Manitoba population that receives health care coverage through the provincial government department Manitoba Health, Seniors and Healthy Living. ${ }^{20}$ These data have been validated and used extensively in health services research. ${ }^{21,22}$

We linked data across multiple data sets using scrambled personal health identification numbers. Several databases were used, including the Drug Program Information Network database, which includes the dispensation date, drug name and medical specialty of the prescriber. This database captures all prescription drugs dispensed by community pharmacies to Manitoba residents regardless of the type of insurance coverage (government-sponsored, private or out-of-pocket). This provides a comprehensive description of nonhospital drug use. The provincial Pharmacare program provides complete coverage for eligible medications for Manitobans after an income-based deductible has been met during the fiscal year. ${ }^{23}$ The Medical Services (physician claims) database provided service date and the International Classification of Diseases, 9th Revision [Clinical Modification] (ICD-9-CM) diagnosis code, and the Discharge Abstract Database provided hospital admission and discharge dates and several diagnoses coded with ICD-9-CM or ICD-10 codes. The Manitoba Health Insurance Registry database provided the number of Manitoba residents at the beginning of each 1-year interval, users' demographic information and the dates of their health care coverage.

\section{Study population and exposure definitions}

We identified pharmaceutical cannabinoid users living in Manitoba during 2004/05 to 2014/15, without age restrictions. We identified prescription dispensations, including nabilone (A04AD11), dronabinol (A04AD10) and nabiximols (N02BG10), by their Anatomic Therapeutic Chemical Classification System code. The number of incident and prevalent users was assessed. We defined incident use as first-time use in the study period, with no record of a previous prescription, since the first year of available data (1995). We defined prevalent use in a specific year as having received at least 1 cannabinoid prescription in that year throughout the study period. We defined region of residence as urban (Winnipeg [population 705 244] and Brandon [48 859]) or rural Manitoba, which includes all other centres (each containing fewer than 16000 people).

We identified the medical conditions of cannabinoid users using algorithms used in previous research ${ }^{24-29}$ that are based on ICD codes from physician claims, hospital admissions and/or use of prescription medications (Supplementary Table A1, Appendix 1, available at www.cmajopen.ca/content/6/4/ E637/suppl/DC1). Medical claims and hospital admissions within 2 years before the first cannabinoid dispensation were used to ensure a recent diagnosis. We chose 11 medical conditions, including the approved indications (multiple sclerosis, HIV/AIDS and cancer, excluding in situ and skin cancer), conditions reported in the literature as possible indications for cannabinoid use (glaucoma, epilepsy, rheumatoid arthritis, osteoarthritis, fibromyalgia and mood/anxiety disorders) ${ }^{4}$ and schizophrenia and substance abuse disorders, as the use of cannabinoids is not recommended in these conditions. ${ }^{30}$ We also assessed users' chronic pain, defined as a minimum of a 180-day supply in at least 2 dispensations of opioids, nonsteroidal anti-inflammatory drug, or acetaminophen and its combinations (excluding psycholeptics and cold preparations) within the year preceding the first cannabinoid prescription. ${ }^{31}$

Last, we determined the medical specialty of prescribers initiating these prescriptions from the deidentified physician identification number reported on the prescription.

\section{Statistical analysis}

We calculated annual prevalence rates of cannabinoid use by dividing the number of prevalent users in a year by the population count on Dec. 31 of that calendar year. We calculated annual incidence rates by dividing the number of first-time users in a year by the population count, excluding prevalent users, in that calendar year. We then calculated the annual incidence rates for subgroups based on age, sex and region of residence. 
We used multivariable Poisson regression analysis, adjusted for age, sex, area of residence and socioeconomic status, to test for temporal trends in the incident and prevalent rates, which were calculated based on aggregate data for each stratum. To account for health coverage that is less than one year, log person-years was included as an offset in the model. Age was included as a categorical variable and was categorized into $\leq 18,19-45,46-64$, and $\geq 65$ years. This categorization was based on guidelines requiring caution when prescribing these agents to children and the elderly and to account for the higher risk of conditions like multiple sclerosis and cancer in older compared to younger adults. Socioeconomic status was categorized into 4 groups based on the Manitoba Centre for Health Policy's Socioeconomic Factor Index, an area-level measure derived from census data. The categorization, according to a validated definition, was based on cut-off points of 1 standard deviation from the mean into high, middle, middlelow and low socioeconomic status. ${ }^{32} \mathrm{~A} p$ value of $\leq 0.05$ was considered significant. We analyzed the data using the SAS software package for Windows, version 9.4 (SAS Institute).

\section{Ethics approval}

This study was conducted in full compliance with the Personal Health Information Act of Manitoba and was approved by the Health Research Ethics Board at the University of Manitoba and the Manitoba Health Information Privacy Committee.

\section{Results}

\section{Prescriptions}

Over the 11-year study period, 76719 cannabinoid prescriptions were dispensed to 5181 unique individuals, 5033 of whom received their first prescription after Apr. 1, 2004. Nabilone accounted for almost all cannabinoid prescriptions dispensed (73 650 [96.0\%]). About one-third (1621 [32.2\%]) of the incident cannabinoid users received only 1 prescription. General practitioners initiated the highest proportion of first prescriptions (2350 [46.7\%]), followed by anesthesiologists/ pain specialists (1299 [25.8\%]). Only 271 prescriptions (5.4\%) were initiated by oncologists.

\section{Incident cannabinoid use}

The mean age of new users was 50.6 (standard deviation $14.7)$ years, and 2934 (58.3\%) were female. The annual incidence rate increased by $413.3 \%$ over the study period, from 12.12 (95\% confidence interval [CI] 12.06-12.19) users per 100000 person-years in 2004/05 to $62.21(95 \%$ CI 62.08-62.35) users per 100000 person-years in 2014/15. The incidence of nabilone use increased by $475.4 \%$, from 10.76 (95\% CI 10.69-10.82) users per 100000 person-years in $2004 / 05$ to 61.91 (95\% CI 61.77-62.05) users per 100000 person-years in 2014/15 (Figure 1). The incident use of both nabiximols and dronabinol was low.

The incident use of cannabinoids by age, sex and region of residence is presented in Figure 2. Over the study period, the incidence rate increased by $242.51 \%$ for females and $141.03 \%$

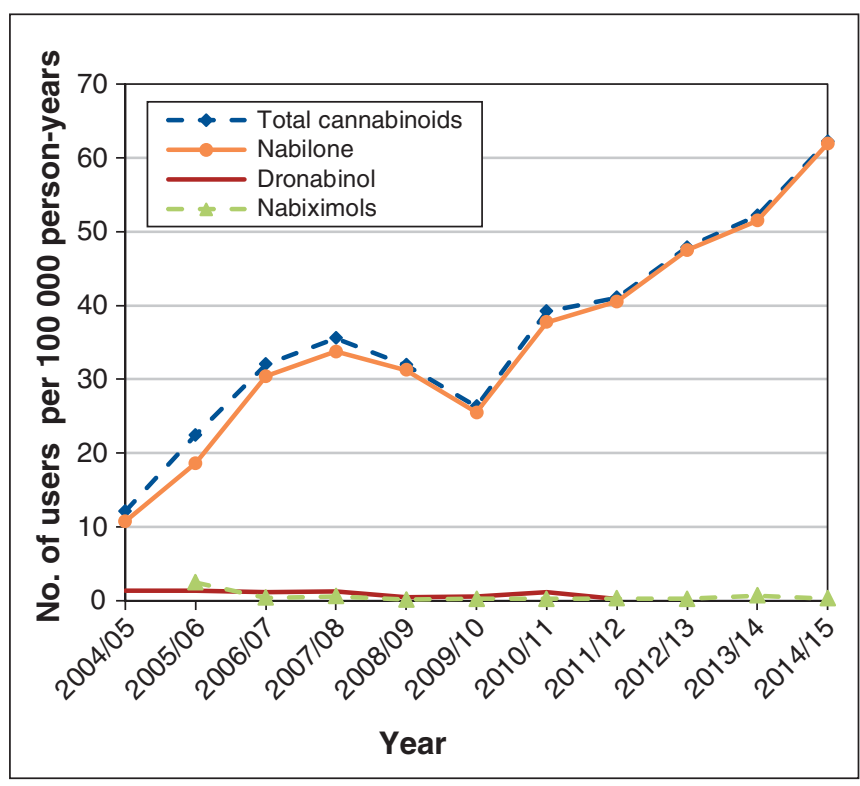

Figure 1: Annual incidence of cannabinoid users in Manitoba, 2004/05-2014/15.

for males (Figure 2, A). The incidence rate for adults aged 46-64 increased by $258.63 \%$ (Figure 2, B). The incidence rate increased by $256.9 \%$ and $86.8 \%$ for those aged 65 years or more and younger adults (age 19-45), respectively. The youngest segment of the population $(\leq 18 \mathrm{yr}$ ) showed the lowest incidence of use. The incident use increased by $154.4 \%$ for residents of urban Manitoba and by $272.1 \%$ for residents of rural Manitoba (Figure 2, C).

\section{Prevalence of cannabinoid use}

The overall rate of use of cannabinoids throughout the study period was 82.4 users per 100000 person-years. The prevalence of cannabinoid use increased by $527.2 \%$ over the study period, from 21.51 users per 100000 people (95\% CI 21.4121.61 ) in $2004 / 05$ to 134.91 users per 100000 people $(95 \%$ CI 134.71-135.11) in 2014/15. The prevalence of nabilone use increased by $642.8 \%$, from 18.01 users per 100000 people (95\% CI $17.93-18.09$ ) in 2004/2005 to 133.77 users per 100000 people (95\% CI 133.57-133.97) in 2014/2015 (Figure 3). The prevalent use of both dronabinol and nabiximols was low throughout the study period.

Incidence and prevalence rates standardized to the Canadian population based on Statistics Canada data in 2016 are reported in Supplementary Figure A2, Appendix 1.

\section{Effect of demographic characteristics on incident and prevalent use}

The sociodemographic characteristics of incident users are presented in Table 1 . After we adjusted for age, sex, area of residence and socioeconomic status, the annual rate of prevalent and incident cannabinoid use increased by 1.15 and 1.09 per 100000 people, respectively. The upward trend was significant for both prevalence and incidence models. The effect of time and user demographic characteristics on use is shown in Table 2. 


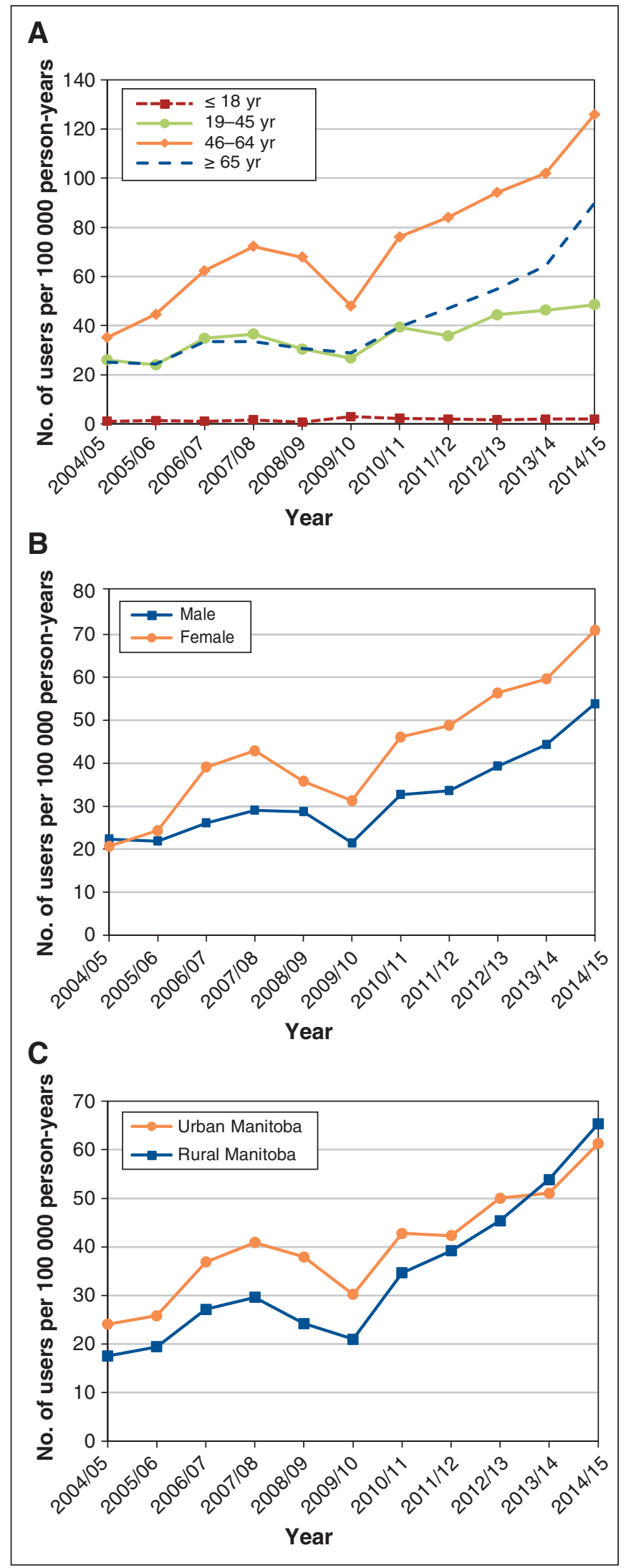

Figure 2: Annual incidence rate of cannabinoid use by age group (A), sex $(B)$ and area of residence $(C)$.

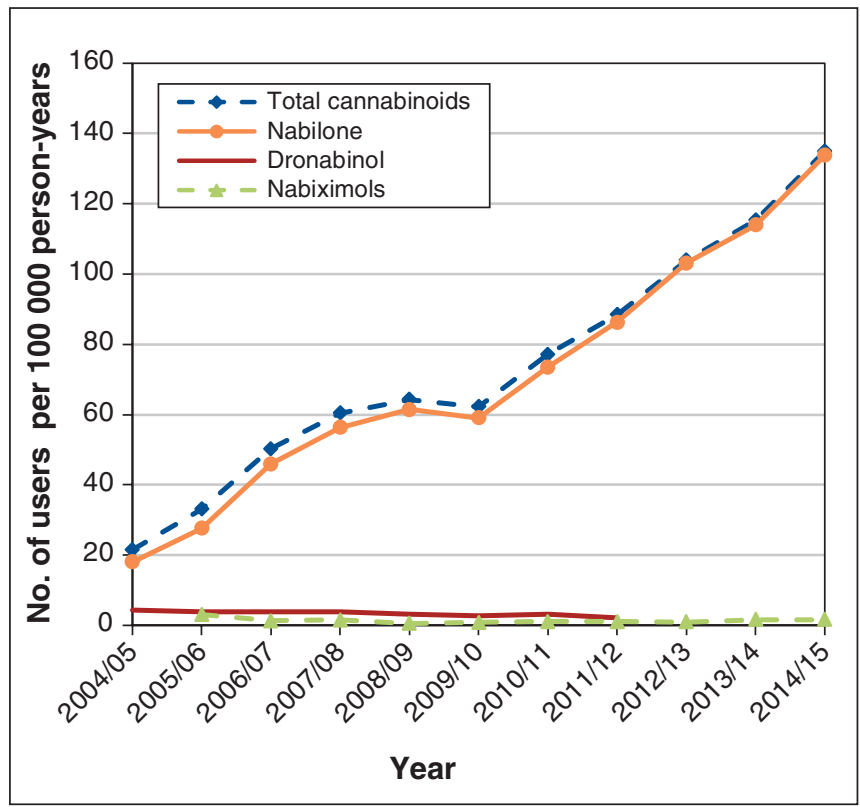

Figure 3: Annual prevalence of cannabinoid use.

\section{Medical conditions of incident users}

We identified 1775 incident cannabinoid users (35.3\%) who had received a diagnosis of fibromyalgia and $1116(22.2 \%)$ with a diagnosis of mood/anxiety disorders (Table 1). The number of users who had a diagnosis of cancer, multiple sclerosis or HIV/AIDS was 917 (18.2\%), 213 (4.2\%) and 51 (1.0\%), respectively. A total of 2540 users (50.5\%) were being treated for chronic pain in the year before their first cannabinoid prescription.

\section{Interpretation}

In this population-based study, we found an increase in the number of pharmaceutical cannabinoid users in Manitoba between 2005/05 and 2014/05, driven almost entirely by nabilone use. In 2009, there was a slight temporary decrease in nabilone use. Considering that there were no alternatives to Cesamet (nabilone) (Valeant Pharmaceuticals International), this decline might be explained by a recall of Cesamet owing to a mislabelling incident identified nationally by Health Canada. ${ }^{33}$

The rates of use were higher for females. This may have been due in part to the fact that conditions such as fibromyalgia, osteoarthritis, and mood/anxiety disorders are more common in women. As expected, the lowest rate of use was among the youngest age group, as the use of cannabinoids in those less than 18 years is not recommended owing to a lack of safety data. The highest rate of use was among people aged 46-64 years, followed by those aged 65 years or more, among whom conditions such as multiple sclerosis and cancer are more prevalent. There was a difference in the rates of use among urban versus rural residents, possibly due to difference in access to and use of health care services.

Most of the medical conditions identified were painrelated conditions such as fibromyalgia, osteoarthritis, cancer 


\begin{tabular}{|c|c|}
\hline Characteristic & $\begin{array}{c}\text { No. }(\%) \text { of users } \\
n=5033\end{array}$ \\
\hline \multicolumn{2}{|l|}{ Age group, yr } \\
\hline$\leq 18$ & $55(1.1)$ \\
\hline $19-45$ & 1698 (33.7) \\
\hline $46-64$ & $2455(48.8)$ \\
\hline$\geq 65$ & $825(16.4)$ \\
\hline \multicolumn{2}{|l|}{ Sex } \\
\hline Female & 2934 (58.3) \\
\hline Male & $2099(41.7)$ \\
\hline \multicolumn{2}{|l|}{ Area of residence } \\
\hline Urban & 3207 (63.7) \\
\hline Rural & $1826(36.3)$ \\
\hline \multicolumn{2}{|l|}{ Socioeconomic status } \\
\hline High & $458(9.1)$ \\
\hline Middle & $1712(34.0)$ \\
\hline Mid-low & $2028(40.3)$ \\
\hline Low & $835(16.6)$ \\
\hline \multicolumn{2}{|l|}{ Medical condition* } \\
\hline Chronic pain & $2540(50.5)$ \\
\hline Fibromyalgia & 1775 (35.3) \\
\hline Mood/anxiety disorder & $1116(22.2)$ \\
\hline Cancer & $917(18.2)$ \\
\hline Osteoarthritis & $662(13.2)$ \\
\hline Substance abuse & $418(8.3)$ \\
\hline Multiple sclerosis & $213(4.2)$ \\
\hline Glaucoma & $169(3.4)$ \\
\hline Rheumatoid arthritis & $155(3.1)$ \\
\hline HIV/AIDS & $51(1.0)$ \\
\hline Schizophrenia & $29(0.6)$ \\
\hline Epilepsy & $27(0.5)$ \\
\hline
\end{tabular}

and multiple sclerosis. Moreover, half of the incident cannabinoid users met the study definition of chronic pain. This pattern of use is consistent with the growing evidence regarding the potential role of cannabinoids in pain management and the safety of chronic opioid use. $4,34,35$ Despite the caution required, we found that $8.3 \%$ of incident users received a diagnosis of substance abuse disorder; this may have been due to prescribers' looking for an alternative to medical cannabis for these patients. The number of users with a diagnosis of schizophrenia was low, as these agents are contraindicated when there is history of psychosis. The proportion of users who met the study definition for the approved indications was low. Moreover, one-third of incident users filled only 1 prescription. This could be a reflection of the real-world
Table 2: Effect of time and sociodemographic characteristics on the prevalence and incidence of cannabinoid use in Manitoba, 2004/05-2014/15

\begin{tabular}{|lcc|}
\hline \multirow{2}{*}{ Variable } & \multicolumn{2}{c|}{ Relative rate $(95 \% \mathrm{Cl})$} \\
\cline { 2 - 3 } & Prevalence & Incidence \\
\hline Change in annual rate & $1.15(1.14-1.16)$ & $1.09(1.08-1.10)$ \\
\hline Female v. male sex & $1.38(1.30-1.46)$ & $1.33(1.23-1.42)$ \\
\hline Age & & \\
\hline$\leq 18$ v. $\geq 65$ & $0.03(0.02-0.04)$ & $0.04(0.03-0.06)$ \\
\hline $19-45$ v. $\geq 65$ & $0.96(0.88-1.06)$ & $0.82(0.74-0.91)$ \\
\hline $46-64$ v. $\geq 65$ & $2.28(2.09-2.49)$ & $1.70(1.53-1.89)$ \\
\hline Urban v. rural residence & $1.17(1.10-1.24)$ & $1.11(1.04-1.19)$ \\
\hline Socioeconomic status & & \\
\hline Low v. high & $0.62(0.55-0.71)$ & $0.79(0.68-0.92)$ \\
\hline Mid-low v. high & $0.73(0.66-0.80)$ & $0.84(0.74-0.95)$ \\
\hline Middle v. high & $0.92(0.89-1.08)$ & $1.06(0.94-1.19)$ \\
\hline Note: Cl = confidence interval. & & \\
\hline
\end{tabular}

effectiveness of these agents; however, the underlying reason for this trend requires further investigation.

The observed overall increase in nabilone use over time may have been related to a general increased interest in medical marijuana, as nabilone represents an option that is easier to access and is covered by the provincial drug program, in contrast to nabiximols. ${ }^{36}$ Furthermore, prescribers may prefer nabilone over raw medical cannabis because it generally causes less euphoria than marijuana and hence is less prone to be abused and because of its consistent, standardized dosing, whereas dosing is highly variable with natural cannabis products. ${ }^{12,37,38}$ However, it is unknown whether this trend will continue after the legalization of recreational cannabis, as some patients may favour purchasing cannabis over a physician visit and filling a prescription. This will be determined by the cost, age limit, quantity limits and other administrative regulations associated with legalization. ${ }^{7}$

\section{Strengths and limitations}

This was an observational, population-based database study that captured nearly every encounter between Manitoba residents with a universal health care system, which allowed for a complete assessment of real-world drug use, without sampling errors. Despite the advantages of observational studies using administrative data, there are recognized limitations, including potential misclassification of medical conditions when using data not intended for research purposes; however, we used algorithms validated in other studies to minimize misclassification. It is impossible to fully determine the intended indication for the use of cannabinoids, which have several potential uses. Thus, even when an associated condition is correctly identified, the clinical indication for cannabinoid use cannot be confirmed. This is especially difficult in chronic pain, as there is no consensus on a validated definition for identifying patients 
with chronic pain with the use of administrative data, despite the fact that numerous painful conditions can be identified. Moreover, other factors that we were not able to examine, such as ethnicity, may have influenced cannabinoid use.

\section{Conclusion}

Incident and prevalent cannabinoid use increased over the study period. Nabilone accounted for most dispensed cannabinoids. Incident use was higher throughout the 11-year period among females and older adults. Pain and pain-related conditions accounted for the highest proportion of possible indications for cannabinoids. These findings provide insight into the use of these agents before policies regarding access to cannabis change. The introduction of recreational marijuana and its legal availability may affect this trend.

\section{References}

1. Cannabis use and disorders. CANSIM table 105-1101. Ottawa: Statistics Canada; 2012. Available: https://www.statcan.gc.ca/pub/13-610-x/cannabis-eng.htm (accessed 2018 Apr. 24).

2. Canadian Alcohol and Drug Use Monitoring Survey. Summary of results for 2011: cannabis use. Ottawa: Health Canada; 2011. Available: https://www.canada. $\mathrm{ca} / \mathrm{en} /$ health-canada/services/health-concerns/drug-prevention-treatment/drug -alcohol-use-statistics/canadian-alcohol-drug-use-monitoring-survey-summary -results-2011.html\#a3 (accessed 2018 Apr. 24).

3. Zuardi AW. History of cannabis as a medicine: a review. Rev Bras Psiquitar 2006;28:153-7.

4. Whiting PF, Wolff RF, Deshpande S, et al. Cannabinoids for medical use: a systematic review and meta-analysis. FAMA 2015;313:2456-73.

5. Allan GM, Jamil C, Danielle R, et al. Simplified guideline for prescribing medical cannabinoids in primary care. Can Fam Physician 2018;64:111-20.

6. Drug Product Database online query. Ottawa: Health Canada; [modified 2018 June 12]. Available: https://health-products.canada.ca/dpd-bdpp/index-eng.jsp (accessed 2017 Dec. 9)

7. Cochrane D. Liberals want to move up pot legalization to avoid Canada Day celebrations. CBC News 2017 Apr. 8. Available: www.cbc.ca/news/politics/ legal-marijuana-date-canada-day-1.4060783 (accessed 2017 Dec. 9).

8. Kalant H. A critique of cannabis legalization proposals in Canada. Int 7 Drug Policy 2016;34:5-10

9. Hajizadeh M. Legalizing and regulating marijuana in Canada: review of potential economic, social, and health impacts. Int 7 Health Policy Manag 2016;5:453-6.

10. Fischer B, Russell C, Sabioni P, et al. Lower-risk cannabis use guidelines: a comprehensive update of evidence and recommendations. Am 7 Public Health 2017;107:e1-12.

11. Romero-Sandoval EA, Kolano AL, Alvarado-Vázquez PA. Cannabis and cannabinoids for chronic pain. Curr Rheumatol Rep 2017;19:67.

12. Ware MA, St Arnaud-Trempe E. The abuse potential of the synthetic cannabinoid nabilone. Addiction 2010;105:494-503.

13. Hazekamp A, Ware MA, Muller-Vahl KR, et al. The medicinal use of cannabis and cannabinoids - an international cross-sectional survey on administration forms. 7 Psychoactive Drugs 2013;45:199-210.

14. Pertwee RG. Cannabis and cannabinoids: pharmacology and rationale for clinical use. Forsch Komplementarmed 1999;6(Suppl 3):12-5.

15. Manzanares J, Julian M, Carrascosa A. Role of the cannabinoid system in pain control and therapeutic implications for the management of acute and chronic pain episodes. Curr Neuropharmacol 2006;4:239-57.

16. Wada JK, Bogdon DL, Gunnell JC, et al. Double-blind, randomized, crossover trial of nabilone vs. placebo in cancer chemotherapy. Cancer Treat Rev 1982;9(Suppl B):39-44.

17. Chan HS, Correia JA, MacLeod SM. Nabilone versus prochlorperazine for control of cancer chemotherapy-induced emesis in children: a double-blind, crossover trial. Pediatrics 1987;79:946-52.

18. Pooyania S, Ethans K, Szturm T, et al. Randomized, double-blinded, crossover pilot study assessing the effect of nabilone on spasticity in persons with spinal cord injury. Arch Phys Med Rehabil 2010;91:703-7.

19. Manitoba Centre for Health Policy [departmental webpage]. Winnipeg: University of Manitoba; 2017. Available: http://umanitoba.ca/faculties/health_ sciences/medicine/units/chs/departmental_units/mchp/(accessed 2017 Nov. 6).

20. Community health assessment guidelines 2009. Winnipeg: Manitoba Health and Healthy Living; 2009. Available: https://www.gov.mb.ca/health/rha/docs/chag. pdf (accessed 2018 Oct. 5).

21. Roos LL, Nicol JP. A research registry: uses, development, and accuracy. 7 Clin Epidemiol 1999;52:39-47.
22. Roos LL, Brownell M, Lix L, et al. From health research to social research: privacy, methods, approaches. Soc Sci Med 2008;66:117-29.

23. About the Manitoba Pharmacare Program. Winnipeg: Manitoba Health, Seniors and Active Living. Available: https://www.gov.mb.ca/health/pharmacare/ (accessed 2018 Oct. 5)

24. Tu K, Wang M, Jaakkimainen L, et al. Assessing the validity of using administrative data to identify patients with epilepsy. Epilepsia 2014;55:335-43.

25. Marrie RA, Fisk JD, Yu BN. Mental comorbidity and multiple sclerosis: validating administrative data to support population-based surveillance. BMC Neurol 2013;13:16.

26. Lix L, Yogendran M, Burchill C, et al. Defining and validating chronic diseases. Winnipeg: Manitoba Centre for Health Policy; 2006.

27. Nosyk B, Colley G, Yip B, et al. Application and validation of case-finding algorithms for identifying individuals with human immunodeficiency virus from administrative data in British Columbia, Canada. PLoSOne 2013;8: $1-7$.

28. Katz A, Martens $\mathrm{P}$, Chateau D, et al. Understanding the bealth system use of ambulatory care patients. Winnipeg: Manitoba Center for Health Policy;2013.

29. Ou Y, Grossman DS, Lee PP, et al. Glaucoma, Alzheimer disease and other dementia: a longitudinal analysis. Ophthalmic Epidemiol 2012;19:285-92.

30. Regier L, Jensen B. Cannabinoids. In: RxFiles drug comparison charts. 11th ed. Saskatoon: Saskatoon Health Region;2017:167.

31. Wertli MM, Reich O, Signorell A, et al. Changes over time in prescription practices of pain medications in Switzerland between 2006 and 2013: an analysis of insurance claims. BMC Health Serv Res 2017;17:167.

32. Concept dictionary and glossary for health services research. Winnipeg: Manitoba Centre for Health Policy; 2012. Available: https://www.umanitoba.ca/ faculties/medicine/units/community_health_sciences/departmental_units/mchp/ resources/concept_dictionary.html (accessed 2018 Oct. 3).

33. Recalls and safety alerts: potential risk of serious adverse health consequences with inadvertent administration of Cesamet (nabilone) capsules $1 \mathrm{mg}$ instead of Trazorel (trazodone hydrochloride) tablets $50 \mathrm{mg}$ due to mislabelling for health professionals. Ottawa: Health Canada; 2009. Available: http:// healthycanadians.gc.ca/recall-alert-rappel-avis/hc-sc/2009/14538a-eng.php (accessed 2018 Apr. 27).

34. Lucas P. Cannabis as an adjunct to or substitute for opiates in the treatment of chronic pain. 7 Psychoactive Drugs 2012;44:125-33.

35. Gomes T, Mamdani MM, Dhalla IA, et al. Opioid dose and drug-related mortality in patients with nonmalignant pain. Arch Intern Med 2011;171: 686-91.

36. Drug formulary lookup. Winnipeg: Manitoba Health; 2017. Available: https:// web22.gov.mb.ca/eFormulary/ (accessed 2017 Dec. 10).

37. Calhoun SR, Galloway GP, Smith DE. Abuse potential of dronabinol (Marinol@). 7 Psychoactive Drugs 1998;30:187-96.

38. Lemberger L, Rubin A, Wolen R, et al. Pharmacokinetics, metabolism and drug-abuse potential of nabilone. Cancer Treat Rev 1982;9(Suppl B):17-23.

Affiliations: College of Pharmacy (Alkabbani, Bugden, Alessi-Severini, Leong), Rady Faculty of Health Sciences; Max Rady College of Medicine (Marrie, Daeninck, Bolton, Sareen), Rady Faculty of Health Sciences, University of Manitoba, Winnipeg, Man.; School of Pharmacy (Bugden), Memorial University of Newfoundland, St. John's, Nfld.

Contributors: Christine Leong conceived the study and supervised the work. Wajd Alkabbani conducted all analyses and drafted the manuscript. Ruth Ann Marrie provided input regarding the methodology and statistical analyses. All of the authors contributed to the study design and interpretation of the data, revised the manuscript for important intellectual content, gave final approval of the version to be published and agreed to be accountable for all aspects of the work.

Funding: This work was supported by funding from the College of Pharmacy, University of Manitoba.

Acknowledgements: The authors thank the Manitoba Centre for Health Policy for use of data contained in the Manitoba Population Research Data Repository under project HIPC 2015/2016-22. The authors also thank and acknowledge Heather Prior and Natalia Dik for retrieving the data.

Disclaimer: The results and conclusions are those of the authors, and no official endorsement by the Manitoba Centre for Health Policy, Manitoba Health, Seniors and Healthy Living, or other data providers is intended or should be inferred.

Supplemental information: For reviewer comments and the original submission of this manuscript, please see www.cmajopen.ca/content/6/4/ E637/suppl/DC1. 